\title{
Business Capability Maps: Current Practices and Use Cases for Enterprise Architecture Management
}

\author{
Pouya Aleatrati Khosroshahi, \\ Florian Matthes, Martin Gernegroß \\ Technical University of Munich \\ \{p.aleatrati, matthes, m.gernegross\}@tum.de
}

\author{
Matheus Hauder \\ Allianz Deutschland AG \\ matheus.hauder@allianz.de
}

\author{
Stefan Volkert \\ BMW Group \\ stefan.volkert@bmw.de
}

\begin{abstract}
This paper provides a state-of-the-art report on the usage of business capability maps in enterprise architecture management. We conducted expert interviews with 25 organizations to reveal the benefits and challenges of capability-based enterprise architecture management and evaluated 14 use cases on the feasibility and benefit of using business capability maps in practice. The results reveal increasing interest and acceptance of the approach in practice and among support organizations.
\end{abstract}

\section{Motivation}

The role of information technology (IT) in organizations has changed over the last decades; technology advances have led to new business opportunities and have forced organizations to undergo fundamental changes in their business models [1], [8], [15]. Challenges are not the only thing at the business strategy level as organizations have to prepare for a mind shift from a structural and operational point of view and undergo fundamental changes in their enterprise architecture (EA) [6]. Considering a recent study by Aleatrati Khosroshahi et al. [4], the role of EA management (EAM) has changed over the last years. The discipline has evolved from an operational practice (e.g., documenting data objects and analyzing processes) to a comprehensive EA optimization activity (e.g., providing transparency and identifying bottlenecks in the application portfolio). These demands call for a powerful tool that provides transparency of the EA from different viewpoints and also considers business demands.

Recent studies have shown that business capability maps (BCMs) have gained great attention in EAM [11], [3]. BCMs help to align IT practices and investments with business demands, support EAM tasks from different viewpoints, and are essential for communication between business and IT stakeholders [5], [12], [19], [22]. Unlike business processes, business capability does not describe a set of activities that needs to be conducted to achieve a concrete result. The EAM standard TOGAF® from the Open Group describes a capability as "an ability that an organization, person, or system possesses." [21]. In other words, a business capability describes a skill or ability that an organization uses to perform its core function. A business capability encompasses and describes all applications, roles, and skills used to provide a business function. It illustrates a loosely coupled group within the organization that aims to provide a specific capability. The BCM is an ordered representation of all business capabilities within the organization. Figure 1 illustrates an example of a BCM. Consider an organization that develops software for insurance companies. Such an organization needs capabilities to develop software ("Development"), run internal processes ("Enterprise Services"), and distribute products ("Sales"). Each of these capabilities includes sub-capabilities. The mapping of EA-related information (e.g., applications) to business capabilities enables the BCM to act as a control instrument and provides transparency about issues within the EA from different viewpoints. However, the use of BCM in EAM is at a very early stage and there is a lack of concrete use cases and visualizations that support EA initiatives.

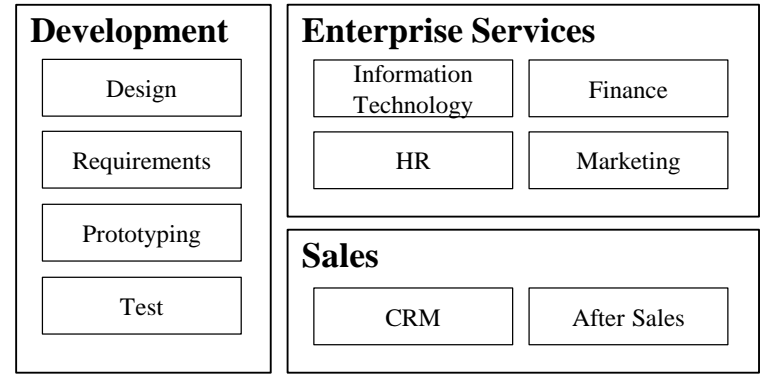

Figure 1: Example of a BCM 
We aim to reveal the role of BCMs in EAM and identify use cases that can be addressed by BCM visualizations. In cooperation with two industry partners, we identified 14 use cases for a capabilitybased EAM and conducted expert interviews with 25 organizations to evaluate the usability of these cases (see Table 1 for details regarding the participants). Our questionnaire includes questions regarding the use of the $\mathrm{BCM}$ within the respective organization to reveal the benefits and challenges of BCMs in practice.

We aim to investigate the following research questions (RQs):

- RQ1: To what extent are BCMs used in practice for EAM?

- RQ2: What are suitable use cases for a capability-based EAM?

The paper is structured as follows. In Section 2, we provide an overview of related work. In Section 3, we illustrate our research approach. An overview of our evaluated use cases and the results of our expert interviews are illustrated in Sections 4 and 5. The paper ends with a discussion of the results in Section 6 and a conclusion and an outlook in Section 7.

\section{Related work}

The TOGAF standard outlines the value of capability-based planning of EAM and states, "from an EA and IT perspective, capability-based planning is a powerful mechanism to ensure that the strategic business plan drives the enterprise from a top-down approach." [21]. The standard elaborates the relationship between business capabilities and EAM and explains the different dimensions that need to be considered when defining business capabilities (i.e., people, processes, and materials). Due to the nature of the framework, TOGAF does not provide results from research activities or name concrete characteristics (e.g., an application of a characteristic for material dimension).

Barroero et al. [5] bridged the gap between the TOGAF concept and missing data, application, and technology architecture by extending the TOGAF meta-model and considering business capabilities. Their contribution considers new architectural artifacts for TOGAF and names changes to the meta-model when considering TOGAF as a capability-centric approach. A further conceptual work by Brits et al. [10] provided a framework on business capability modeling and elaborated guidelines on how to differentiate between types of business capabilities. Their contribution differentiates between functional, integral, dynamic, and strategic capabilities and names critical information that should be analyzed in a capability-based setup (e.g., customers, suppliers, operational business processes, and strategic objectives).

Other researchers distanced themselves from conceptual research activities and investigated concrete methodologies for a capability-based EAM. Freitag et al. [11], for instance, provided a methodology to identify dependencies between business capabilities and evaluated their approach within a telecommunication company. Klinkemüller et al. [13] provided a visualization methodology by introducing a three-dimensional visualization of business capabilities that considers vertical and logical dependencies between business capabilities.

Concrete applications of business capabilities in the EAM domain were provided by Keller [12] who named concrete use cases (e.g., investment decisions, IT/business alignment, and outsourcing decisions), although visualizations are missing.

There are further investigations on how BCM can add value to EAM [7], [20], [23]. A state-of-the-art contribution that outlines the challenges, benefits, and current status of BCMs in organizations is missing. Moreover, our contribution aims to identify concrete use cases and visualizations of BCMs for EAM.

To have a first sample of BCM use cases that could support architectural decisions from different viewpoints, our literature review considers the identification of metrics that affect such decisions. The review considers technical (e.g., lifecycle of applications and interfaces) and organizational (e.g., compliance issues and costs) metrics. Moreover, we considered the needs and challenges within EAM practice that could be addressed with BCM use cases.

Since these contributions investigate other disciplines of EAM and information systems research (e.g., application portfolio management and complexity of IT), rather than BCM, the results of the identified metrics and use cases are not detailed in this section. Section 5 provides an overview of the evaluated use cases and, for each description, includes the sources on which the definition is based.

Our literature review reveals that several activities have already taken place to analyze the use and benefits of BCMs for EAM practice. Related work also elaborates how the TOGAF framework could be enriched with a capability-based view, how to differentiate between types of business capabilities, and what kind of information could be analyzed with this view. However, a state-of-the-art analysis that evaluates the current challenges and benefits for large organizations is still missing. Moreover, related work outlines the value of BCMs, but does not provide concrete use cases for EAM practice. 


\section{Research approach}

We conducted this research in collaboration with two organizations. One organization is an automotive company, headquartered in Europe with approximately 120,000 employees. The other organization is an insurance company, headquartered in Europe with approximately 30,000 employees. Both organizations have profound knowledge about BCMs and their use in EAM and assisted us in defining our research scope and possible use cases. Group discussions with these organizations helped us to ensure that a wide range of relevant use cases was considered for our expert interviews. Hence, the use cases considered in our expert interviews are based on input from research (literature review) and relevant topics from practice. The evaluation of the use cases and the identification of current challenges and benefits of a capability-based EAM are based on expert interviews with 25 organizations. We aimed to have intensive discussions with practitioners who have profound knowledge about BCMs. Thus, we used a qualitative research approach, rather than a quantitative one. The research took place from September 2016 to June 2017 and is illustrated in Figure 2. An overview of the interview partners is illustrated in Table 1.

Identify problem and motivate: In the first phase, we defined the scope of our research, concretized our RQs, and conducted a literature review. The activities were conducted in strong collaboration with our research partners. We had several group discussions with enterprise architects at the respective organizations.

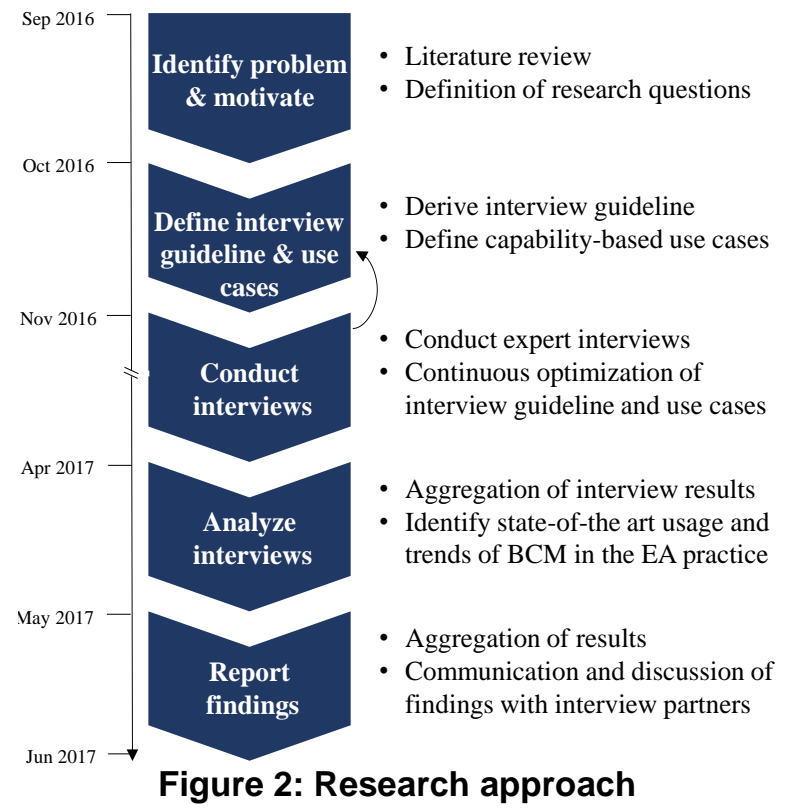

Both organizations were working on large transformations within their enterprises and stated that the BCM was being used as the central artifact to steer and orchestrate the EA transformation. However, they also stated that definitions of concrete use cases for a capability-based EAM are still missing. On the basis of this feedback, we sharpened our RQs and conducted a literature review, considering various journals and online catalogs (i.e., ScienceDirect, IEEE Xplore Digital Library, ACM Digital Library, and Google Scholar). Related work about BCM is illustrated in Section 2 while EA metrics that need to be considered for the use cases are shown in Section 5.

Define interview guideline and use cases: Based on our findings from the literature review and the feedback from the research partners, we defined an interview guideline and use cases for the evaluation.

The interview guideline is divided into three parts (1. general information about interview partner and company, 2. benefits and challenges of BCM, and 3 . evaluation of use cases) and follows a semi-structured approach to discuss a wide range of aspects.

Table 1: Interview partners

\begin{tabular}{|c|l|r|c|}
\hline ID & \multicolumn{1}{|c|}{ Industry } & $\begin{array}{r}\text { Head } \\
\text { count ( } \mathbf{( k )})\end{array}$ & $\begin{array}{c}\text { Experience } \\
\text { EAM }(\mathbf{y r s})\end{array}$ \\
\hline Org.01 & Insurance & 30 & 5 \\
\hline Org.02 & Automotive & 120 & 10 \\
\hline Org.03 & Energy & 60 & 6 \\
\hline Org.04 & Financial Services & 60 & 12 \\
\hline Org.05 & Financial Services & 13 & 6 \\
\hline Org.06 & Insurance & 44 & 8 \\
\hline Org.07 & Logistic & 500 & 10 \\
\hline Org.08 & Chemicals & 65 & 7 \\
\hline Org.09 & Media & 3.5 & 4 \\
\hline Org.10 & Chemicals & 17 & 4 \\
\hline Org.11 & Telecom & 225 & 10 \\
\hline Org.12 & Information Tech. & 380 & 18 \\
\hline Org.13 & Consumer Goods & 57 & 3 \\
\hline Org.14 & Telecom. & 150 & 25 \\
\hline Org.15 & Insurance & 10 & 8 \\
\hline Org.16 & Conglomerate & 350 & $>20$ \\
\hline Org.17 & Financial Services & 6 & 10 \\
\hline Org.18 & Financial Services & 0.5 & 3 \\
\hline Org.19 & Conglomerate & 375 & 4 \\
\hline Org.20 & Financial Services & 11 & 16 \\
\hline Org.21 & Information Tech. & 85 & 10 \\
\hline Org.22 & Conglomerate & 150 & 7 \\
\hline Org.23 & Financial Services & 3.5 & 10 \\
\hline Org.24 & Public Sector & 1 & 7 \\
\hline Org.25 & Consumer Goods & 18 & 6 \\
\hline & & & \\
\hline
\end{tabular}


Conduct interviews: We interviewed 25 organizations ("Org.<number>" in Table 1) from Germany and Switzerland. The participants were identified on the basis of contacts from previous research projects and postings on social media platforms (e.g., LinkedIn).

Each interview lasted approximately one hour and was conducted via phone call or in person. We only considered people for our interviews that had profound knowledge about EAM and stated that further insights about BCM support their EAM strategy. The experts received the interview guideline about one week before the interview. After each interview, we reviewed our interview guideline and updated our questions based on the gathered information. Our interview partners operated in various branches, which indicates that BCMs do not have a branch focus.

Analyze interviews: After the completion of all interviews, the gathered information was aggregated and analyzed for findings. The analysis of the interview follows the matrix-based method by Webster and Watson [23]. The findings were aggregated to statistics that illustrate the general use of BCMs in practice (see Section 4) and implemented use cases (see Section 5).

Report findings: The findings were aggregated and documented in a presentation and communicated to the interview partners.

\section{Use of BCMs}

\subsection{Current use of $\mathrm{BCM}$}

Of the 25 surveyed organizations, 23 use BCMs. Most of them use BCM to strengthen the communication between the management and IT, achieve transparency in the EA, and develop target architectures that consider business characteristics. The two organizations that do not use BCM provided clear reasons: a lack of understanding and acceptance by stakeholders and missing data.

In our interviews, we asked the participants how many years they have been using BCMs and whether the $\mathrm{BCM}$ is used for strategic purposes (e.g., EA investments and target architecture) or operational purposes (e.g., analysis of dependencies and number of applications in each capability).

Figure 3 provides an aggregated overview of the answers regarding the years of use. Figure 4 provides an overview of how many organizations use BCMs for strategic and how many do so for operational purposes.

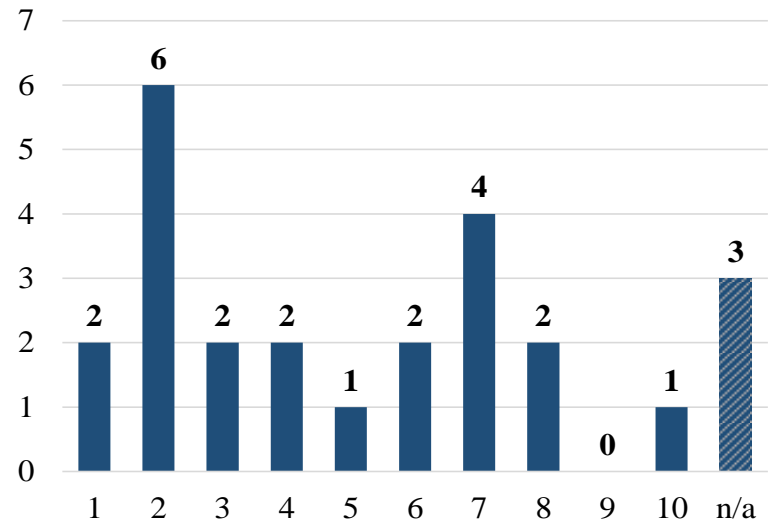

Figure 3: Years of use of BCM

There are two peaks in Figure 3, one at two years of experience, the second at seven years. The peak at two years underlines that using BCMs within EAM activities is still in the early stages in some organizations. The second peak at seven years can be explained by the growing popularity of the EAM discipline in the interviewed organizations: on average, the interviewed organizations have about eight years of experience with EAM, so a considerable number of organizations introduced BCMs in their EAM activities very quickly.

$92 \%$ of the interviewees stated that BCMs are used for strategic purposes and $76 \%$ said that they are used as operational decisions support. Although our results show that BCMs serve mostly for strategic purposes, the statistic reveals the multifacetedness of BCMs in organizations. A correlation between the experience level and the type of use could not be proven.

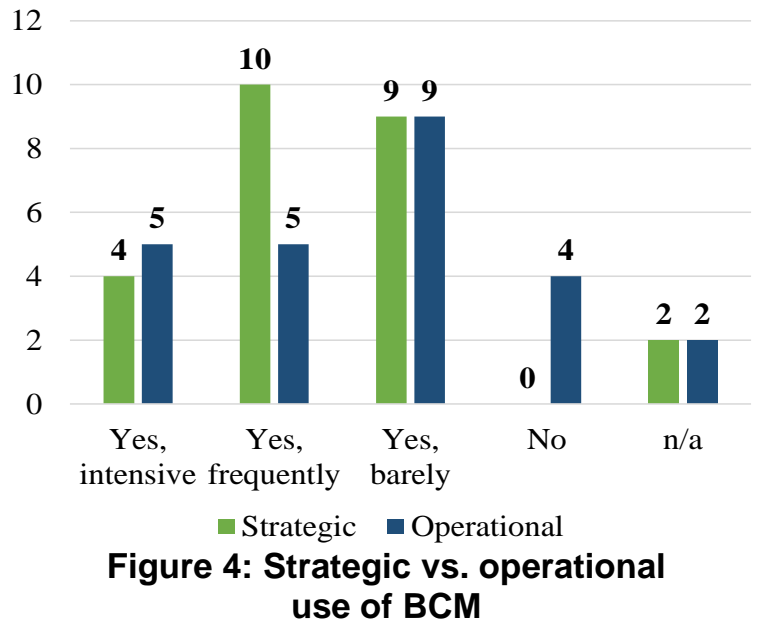

One question in the interviews asked which data are mapped to single business capabilities. On the basis of our literature review and group discussions with our research partners, we asked about the mapping of ten information objects illustrated in Table 2 that consider 
architectural (e.g., applications and technologies), business-related (e.g., projects and business demands), and other metrics that might affect EA decisions. The results show that most of the interviewed organizations map their applications, responsibilities, and processes on the BCM. Applications still have a major role within architectural decisions; they are measurable and further data objects can be assigned to these (e.g., costs, incident tickets, and interfaces), which enable the analysis of the EA from different viewpoints. The results also show that practitioners devote much attention to assigning responsibilities, also called capability "owner," during the interviews.

Some interviewees provided us with additional information mapped to their BCM, including capability priority, strategic direction, interfaces, business organizations, business functions, and locations/regions (named by three organizations).

Table 2: Information mapped to BCMs

\begin{tabular}{|c|c|c|c|c|c|c|c|c|c|c|}
\hline & 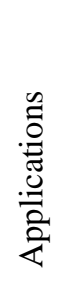 & 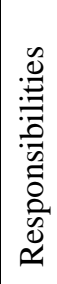 & $\begin{array}{l}0 \\
0 \\
\infty \\
0 \\
0 \\
0 \\
0 \\
0\end{array}$ & $\begin{array}{c}\stackrel{0}{0} \\
\frac{0}{0} \\
0 \\
0\end{array}$ & $\begin{array}{l}\tilde{\mathscr{O}} \\
0 \\
0\end{array}$ & 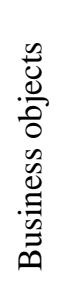 & 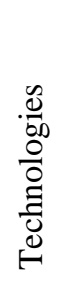 & 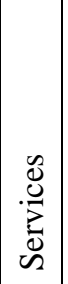 & 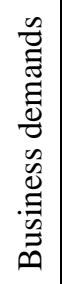 & 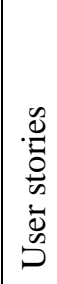 \\
\hline Org.01 & $\mathrm{X}$ & & $\mathrm{X}$ & $\mathrm{X}$ & $\mathrm{X}$ & $\mathrm{X}$ & $\mathrm{X}$ & $\mathrm{X}$ & $\mathrm{X}$ & $\mathrm{X}$ \\
\hline \begin{tabular}{|l|} 
Org.02 \\
\end{tabular} & $\mathrm{X}$ & $\mathrm{X}$ & $\mathrm{X}$ & & $\mathrm{x}$ & $\mathrm{X}$ & & $\mathrm{x}$ & $\mathrm{X}$ & $\mathrm{X}$ \\
\hline Org.03 & $\mathrm{X}$ & $\mathrm{X}$ & $\mathrm{X}$ & $\mathrm{X}$ & & $\mathrm{X}$ & $\mathrm{X}$ & & & \\
\hline \begin{tabular}{|l|} 
Org.04 \\
\end{tabular} & $\mathrm{x}$ & $\mathrm{x}$ & $\mathrm{x}$ & $\mathrm{x}$ & & & $\mathrm{X}$ & & $\mathrm{x}$ & \\
\hline Org.05 & $\mathrm{x}$ & $\mathrm{X}$ & $\mathrm{x}$ & & & $\mathrm{X}$ & & & & $\mathrm{X}$ \\
\hline Org.06 & $\mathrm{x}$ & $\mathrm{x}$ & $\mathrm{x}$ & $\mathrm{x}$ & & & & $\mathrm{X}$ & & \\
\hline Org.07 & $\mathrm{x}$ & $\mathrm{X}$ & $\mathrm{X}$ & $\mathrm{X}$ & & $\mathrm{X}$ & & & & \\
\hline \begin{tabular}{|l|} 
Org.08 \\
\end{tabular} & $\mathrm{X}$ & $\mathrm{X}$ & $\mathrm{X}$ & & $\mathrm{x}$ & & & $\mathrm{X}$ & & \\
\hline Org.09 & $\mathrm{X}$ & $\mathrm{X}$ & $\mathrm{X}$ & & $\mathrm{x}$ & $\mathrm{X}$ & & & & \\
\hline \begin{tabular}{|l|} 
Org.10 \\
\end{tabular} & $\mathrm{x}$ & & & $\mathrm{x}$ & & $\mathrm{x}$ & $\mathrm{x}$ & & & $\mathrm{x}$ \\
\hline \begin{tabular}{|l|} 
Org.11 \\
\end{tabular} & $\mathrm{X}$ & & $\mathrm{X}$ & & $\mathrm{X}$ & & & & $\mathrm{X}$ & \\
\hline Org.12 & $\mathrm{X}$ & $\mathrm{X}$ & & $\mathrm{x}$ & & & $\mathrm{x}$ & $\mathrm{X}$ & & \\
\hline \begin{tabular}{|l|} 
Org.13 \\
\end{tabular} & $\mathrm{X}$ & $\mathrm{x}$ & $\mathrm{x}$ & & $\mathrm{x}$ & & & & & \\
\hline Org.14 & $\mathrm{X}$ & $\mathrm{X}$ & $\mathrm{X}$ & & & & & & $\mathrm{X}$ & \\
\hline Org.15 & $\mathrm{X}$ & $\mathrm{x}$ & & $\mathrm{x}$ & & & $\mathrm{X}$ & & & \\
\hline Org.16 & $\mathrm{X}$ & & $\mathrm{X}$ & & & & & $\mathrm{X}$ & $\mathrm{x}$ & \\
\hline \begin{tabular}{|l|} 
Org.17 \\
\end{tabular} & & & & $\mathrm{x}$ & & $\mathrm{x}$ & & & & $\mathrm{x}$ \\
\hline Org.18 & $\mathrm{X}$ & $\mathrm{X}$ & & $\mathrm{X}$ & & & & & & \\
\hline Org.19 & $\mathrm{X}$ & & & $\mathrm{X}$ & $\mathrm{X}$ & & & & & \\
\hline \begin{tabular}{|l|} 
Org.20 \\
\end{tabular} & $\mathrm{X}$ & & & & $\mathrm{x}$ & & & & & \\
\hline \begin{tabular}{|l|} 
Org.21 \\
\end{tabular} & $\mathrm{X}$ & $\mathrm{X}$ & & & & & & & & \\
\hline \begin{tabular}{|l|} 
Org.22 \\
\end{tabular} & $\mathrm{x}$ & & & & & & $\mathrm{x}$ & & & \\
\hline \begin{tabular}{|l|} 
Org. 23 \\
\end{tabular} & $\mathrm{X}$ & & & $\mathrm{X}$ & & & & & & \\
\hline \begin{tabular}{|l|} 
Org.24 \\
\end{tabular} & & & & & & & & $\mathrm{X}$ & & \\
\hline Org.25 & $\mathrm{n} / \mathrm{a}$ & $\mathrm{n} / \mathrm{a}$ & $\mathrm{n} / \mathrm{a}$ & $\mathrm{n} / \mathrm{a}$ & $\mathrm{n} / \mathrm{a}$ & $\mathrm{n} / \mathrm{a}$ & $\mathrm{n} / \mathrm{a}$ & $\mathrm{n} / \mathrm{a}$ & $\mathrm{n} / \mathrm{a}$ & $\mathrm{n} / \mathrm{a}$ \\
\hline Total & 22 & 14 & 13 & 12 & 8 & 8 & 8 & 7 & 6 & 5 \\
\hline
\end{tabular}

\subsection{Challenges of BCM}

Although we outlined the benefits of BCMs for EAM, our results revealed the novelty of the concept. Thus, we asked the respondents to describe challenges that arise when defining and communicating the BCM to stakeholders within the organization. Figure 6 provides an overview of the most mentioned challenges. A lack of understanding is the most mentioned challenge (by $64 \%$ of organizations); business capabilities differ from business process thinking and require a mind shift within the organization. Our results show that this poses a hurdle, underpinned by missing acceptance (by 44\% of organizations) and lack of management support (28\%). Operative challenges (e.g., creation efforts) were also mentioned, which show that the implementation of a capability-based EAM calls for major efforts.

IT

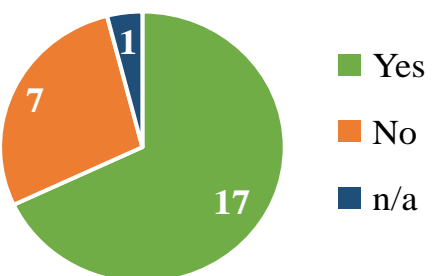

Business

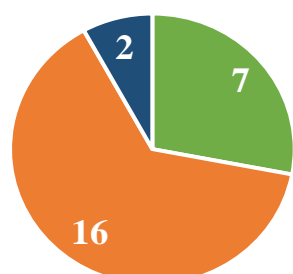

Figure 5: Sufficient communication of BCM

A further question evaluated whether the BCM is sufficiently communicated to the IT and business departments. The results are illustrated in Figure 5. Both departments require additional communication techniques, while business stakeholders call for more attention; $64 \%$ of the organizations stated that their business stakeholders are not familiar with their BCM. Most of the interviewees stated that business stakeholders do not see the added value of the concept. A third question investigated communication methods of the BCM with the following result (relative frequency in brackets): intranet/wiki (92\%), training/workshops (64\%), print (44\%), and lectures $(32 \%)$.

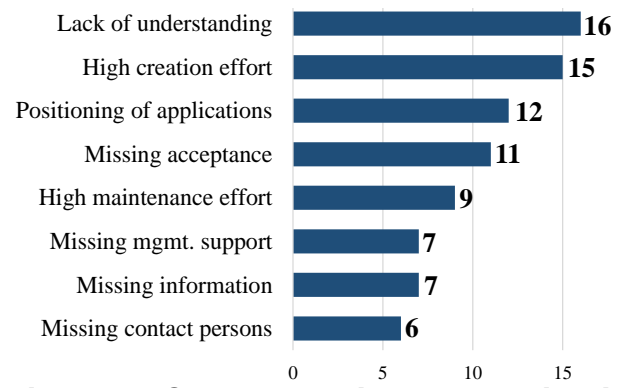

Figure 6: Challenges in communicating $\mathrm{BCMs}$ in EAM 


\section{Use Case evaluation}

\subsection{Overview of use cases}

We evaluated 14 use cases on their usability. The use case is described in the prepared interview guideline for the interview partners. Figure 7 and 8 illustrate two use cases (application lifecycle and capability spanning applications, respectively) in detail. All use cases assume a full mapping of applications to their supported business capabilities.

- Application lifecycle: This use case addresses the retirement dates of applications. The age of an application is a complexity driver for EAs (e.g., high amount of customization and extended support costs); thus, applications nearing retirement should be addressed early by enterprise architects [2], [17] [24]. Heat mapping (red, yellow, and green) in Figure 7 indicates which applications call for further attention from a lifecycle point of view. A business capability that is supported by applications that lose software support might lead to unnecessary costs due to extended support costs or security issues due to missing updates from software providers.

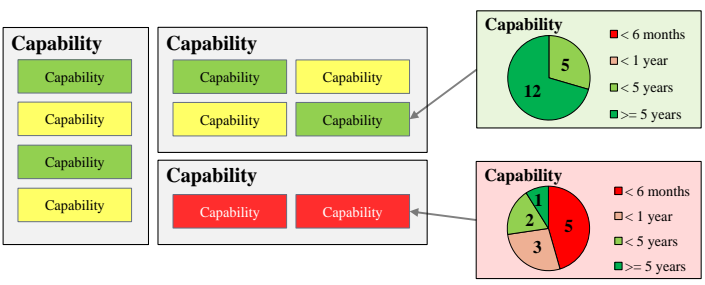

Figure 7: Application lifecycle

- Capability spanning applications: As mentioned by our industry partners, the EA should align with the BCM. Applications that support multiple business capabilities indicate unnecessary dependencies within the EA and act as complexity drivers [12], [24]. Heat mapping at the business capability level indicates whether the capability is supported by a high (red), medium (yellow), or low (green) number of capability spanning applications.

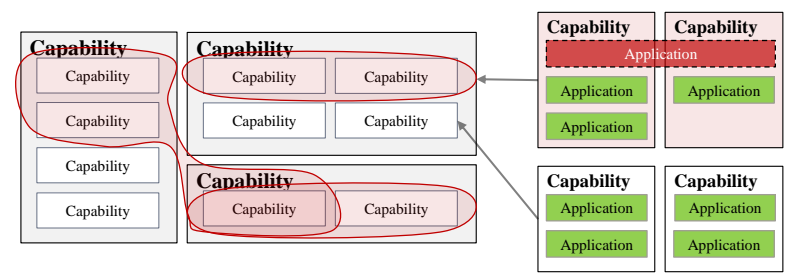

Figure 8: Capability spanning applications
The following enumeration provides an overview of the additional use cases that were evaluated during our interviews. Each use case can be illustrated on a BCM (like Figure 7 and 8) using heat maps.

- Application extended support: Both industry partners mentioned that applications that have already run out of support by the software vendor produce extensive costs for extended support. A heat map on business capabilities indicate the amount of applications that have run out of support $($ red $=$ high, yellow $=$ medium, green $=$ low $)$.

- Cost vs. user count ratio: In the literature, the number of users indicates the importance of an application because a failure of an application might hinder users in their daily work [14], [24]. Other researchers argued that the number of users indicates the complexity of an application (e.g., number of business requirements and incident tickets) [2], [17]. However, in this case, we evaluate the number of users from a different viewpoint. Applications with a low number of users but high operating costs should be evaluated on their need. Decomposition can save high operating costs with a small business impact. Color-coding at the capability level indicates the ratio of operating costs and number of users on average.

- Cloud candidates: Our industry partners mentioned that the identification of cloud candidates takes high priority in their organization. Color-coding at business capability level indicates the amount of applications (percentage) that are operated in the cloud.

- Compliance issues: Based on the covered business requirements, each business capability meets different compliance criteria - e.g., regulatory requirements and security policies [12], [16]. Heat mapping indicates the number of compliance issues within the business capability and provides transparency for project definitions.

- Capability dependencies: The literature classifies interfaces (or any type of dependencies between applications) as complexity drivers in EA [2], [9], [14], [16], [17], [24]. Based on the mapping of applications to business capabilities, dependencies between two applications indicate dependencies between business capabilities. Based on the feedback of our industry partners, business capabilities should be highly decoupled and, thus, avoid a high number of dependencies to other business capabilities. Heat mapping at the business 
capability level illustrates the number of dependencies to other business capabilities.

- Harmonization potential: Functional redundancy is an indicator for complexity of the EA and avoidable IT costs [17], [18]. Redundancies of applications within a business capability indicate harmonization potentials.

- IT costs: The literature already discussed intensely that the amount of IT costs (e.g., for releases, upgrades, and operating) are a crucial factor for EA decisions [14], [17], [20], [24]. A heat mapping should indicate the average operating costs for each application within a business capability.

- Projects: Our industry partners mentioned that a mapping of running EA projects within business capabilities supports the long-term planning of IT budget and projects. A heat mapping indicates the number of EA projects or average project costs in each business capability.

- Business impact: Based on discussions with our industry partners, a measure to determine the business impact - or business value [16], [20] - of a business capability supports the long-term planning of the EA and projects. A heat mapping indicates the importance of a business capability.

- Agile team organization: One industry partner mentioned that the BCM is used in the EAM to evaluate the staffing of their project teams; their project team organization strongly aligns with agile software engineering principles. The definition of user stories is one essential process in their agile approach. These user stories are mapped to the addressed business capabilities and provide advice as to whether a project team addresses one or several business capabilities. Heat mapping indicates missing or incorrect staffing within a business capability.

- Infrastructure components: The number and diversity of infrastructure components within EAs is a well-studied complexity driver [17], [18], [24]. Heat mapping indicates the number of infrastructure components, on average, for each application within a business capability.

- Infrastructure components - extended support: Infrastructure within an extended support indicates old technologies and avoidable costs for the extended support [9], [18], [24]. Heat mapping indicates the number of infrastructure components that are on extended support, on average, for each application (percentage) within a business capability.

We evaluated whether the interviewed organizations have already implemented (or plan on implementing) the use cases and the benefit of each use case (transparency or deriving of concrete actions). We also asked for the feasibility of each use (effort for implementation in an EA tool, gathering information to realize use cases, etc.). On the basis of the benefit and the feasibility, we calculated a benefit/feasibility rating for each use case. The results are illustrated in Section 5.2 .

\subsection{Implementation of use cases in practice}

In every interview, we elaborated whether the interviewed organization plans to implement or has already implemented the use cases. The results are illustrated in Figure 9. Although the results show a fragmented picture, some use cases deserve higher attention. Use cases that aim to decrease hidden complexity in EAs (e.g., harmonization potential and capability spanning applications) are highly identified in EAM practice (or planned practice). Use cases that support EAM decisions based on expenses (e.g., projects and IT costs) are also in use or planned for implementation. The results also show that many use cases are in the "planned" status, which proves the novelty of capability-based EAM.

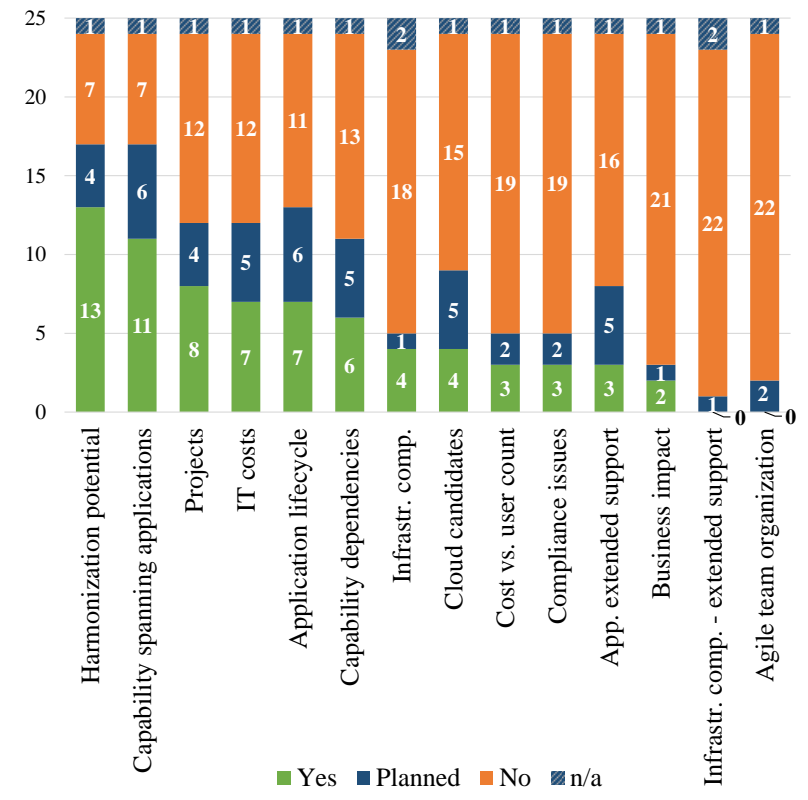

Figure 9: Implementation of use cases 
Two use cases (i.e., infrastructure component extended support and agile team organization) are implemented by none of the organizations. The experts mentioned that these use cases would not bring any benefit or new insights for EA optimization. Decisions are mainly influenced by costs, projects, and complexity that are driven by unnecessary heterogeneity or redundancy. Most of the interviewed organizations obtain infrastructure components from external service providers; thus, these aspects have no impact on their EA.

The experts were asked to estimate for each use case the benefit $(1=$ very low, $2=$ low, $3=$ high, $4=$ very high) and the feasibility for implementation (same scale as benefit) in terms of data availability/quality, effort for implementation, etc. We used the answers to calculate a rating for each use case. The result is illustrated in Figure 10. Each use case is categorized into one of four quadrants.

- Upper right: high benefit/high feasibility

- Lower right: high benefit/low feasibility

- Lower left: low benefit/low feasibility

- Upper left: low benefit/high feasibility

The size of the bubble indicates how many organizations have implemented or plan to implement the use case. The use cases in the upper-right quadrant indicate high attraction in the EAM community; they are feasible for implementation and bring high benefit. In this quadrant, we identify seven use cases: capability spanning applications, harmonization potential, projects, capability dependencies and partly IT costs, cloud candidates, and application lifecycle. The lowerleft quadrant illustrates use cases that are neither feasible nor bring benefit for EAM practice (e.g., agile team organization, applications with extended support). The other use cases either do not bring high benefit or are not feasible for implementation.

The most cited reason for low feasibility is low data quality or missing information. Given the number of companies actively using or planning to implement each use case (e.g., 13 in application lifecycle) as well as the calculated benefit/feasibility rating (see the calculation of rating in the bottom of Figure 10), a correlation between these values was proven (Pearson correlation coefficient: 0.92).

In general, a capability-based EAM is considered a novel approach by practitioners, but more experienced users of BCM or experts already consider feasibility and benefit in their use case implementation agenda. This indicates a strong maturity level, since these organizations have learned to concentrate on meaningful use cases.

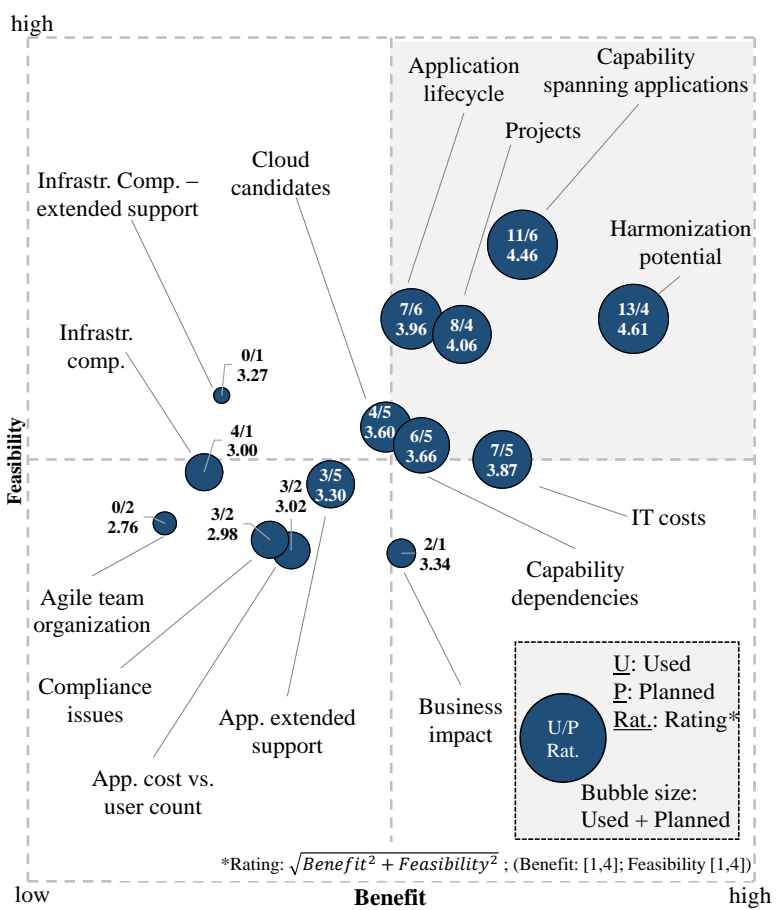

Figure 10: Use case rating (benefit/feasibility)

\section{Discussion}

In our first RQ, we asked to what extent BCMs are used by EAM practitioners. According to our interviews with 25 organizations from different industries, our results reveal that the concept is novel in the EAM community and most of the interviewed organizations started considering BCMs in their EAM two years ago (see Figure 3). However, the diversity of the interviewed organizations (in industry and size in terms of employees) reveals that the concept has a broad attraction in practice. Organizations use BCM for strategic and operational purposes (see Figure 4). However, the experts also mentioned major challenges that they must meet; $64 \%$ of the participants explained that the concept is hard to understand by stakeholders in organizations and $60 \%$ mentioned the high amount of effort in defining the BCM in their organizations (see Figure 6). One further challenge is the communication of the BCM to non-EAM stakeholders (e.g., other IT and business department). Although $68 \%$ of the experts mentioned that the BCM is sufficiently communicated to their IT departments, the results show major challenges in terms of sufficient communication to the business departments $(64 \%$ of participants mentioned insufficient communication). 
In our second RQ, we evaluated which concrete use cases are considered by the experts as useful for EAM practice. We defined 14 use cases with two industry partners in advance and discussed their feasibility and benefit in the interviews. Moreover, we elaborated which of the mentioned use cases are already implemented, planned, or out of scope by the experts. The results in Figure 9 show a fragmented picture; although some use cases reveal complexity in the EA (e.g., capability spanning applications and harmonization potential), the results also show that some organizations are still at the very beginning (many use cases are "planned"). However, the results of our study also show that organizations consider both the feasibility and benefit in their use case choice; the results in Figure 10 show that there are dedicated use cases that are feasible for implementation, bring high benefit, and raise high attraction in the EAM community (correlation between the implementation plan in Figure 9 and the benefit/feasibility rating in Figure 10). The results also show that there are use cases that are neither planned for implementation nor bring any benefit or are not feasible (e.g., agile team organization and infrastructure component - extended support). Although the research provides further insights into BCM for EAM research, there are some limitations within the results. Our results provide transparency about challenges when defining a BCM in large organizations, whereas clear solutions are not provided yet (e.g., methodologies and software support to decrease effort). Our results do not provide any insights on how organizations approach BCM definition and what must be considered when starting respective activities (e.g., how to define business capabilities, how to approach barriers of acceptance in organizations). Moreover, the results derive and evaluate concrete use cases, but do not provide concrete information as to how heat mapping should be conceptualized within each use case.

\section{Conclusion}

In this research, we aim to reveal the status of BCMs in EAM practice and to identify and evaluate concrete use cases for a capability-based EAM approach. We followed a qualitative research approach and conducted expert interviews with 25 organizations among various industries. The evaluated use cases were defined in strong collaboration with two European-based organizations from the automotive and the insurance industry. Each interview took approximately 1 hour and was conducted in person or via telephone. Our results show that BCMs are at the very early stages in EAM practice but are highly attractive in the community. Of the 25 organizations, 23 have BCM on their EAM agenda and are planning on implementing or have already implemented use cases for a capability-based EAM. However, the results also show that the experts face major challenges (see Figure 6) considering organizational and conceptual challenges.

Our results also show that there are dedicated use cases that gain more attraction (e.g., capability spanning applications and harmonization potential) than others (e.g., agile team organization and applications with extended support). This positioning is based on a benefit/feasibility rating that was evaluated for each case during the interviews.

Our results shed light on the BCM for EAM research. It provides a state-of-the-art report and illustrates the current benefits and challenges. The results can be used in practice for selection of use cases. Moreover, the results act as a signal for those organizations that are still evaluating whether they should use BCMs in their EAM practice or not; a large number of organizations already considered this approach in their EAM. Further research should conceptualize the heat mapping of the use cases and derive a clear methodology on how to define and communicate the BCM approach in large organizations. Furthermore, clustering of organizations based on attributes, such as size, industry sector, or experience with EAM, could be evaluated to make more precise recommendations for particular use cases.

\section{References}

[1] M. M. Al-Debei and D. Avison, "Defining the Business Model in the New World of Digital Business", in: Proceedings of the 14th Americas Conference on Information Systems (AMCIS), Toronto, Canada, 2008, pp. 1-11.

[2] P. Aleatrati Khosroshahi, J. Beese, and S. Aier, "What Drives Application Portfolio Complexity?", in: 18th IEEE Conference on Business Informatics (CBI), Paris, France, 2016.

[3] P. Aleatrati Khosroshahi, M. Hauder, A.W. Schneider, and F. Matthes, "Enterprise Architecture Management Pattern Catalog V2", Technical Report 1.0, Technical University of Munich, Germany, 2015.

[4] P. Aleatrati Khosroshahi, M. Hauder, and F. Matthes, "Analyzing the Evolution and Usage of Enterprise Architecture Management Patterns", in: 22th Americas Conference on Information Systems (AMCIS), San Diego, USA, 2016. 
[5] T. Barroero, G. Motta, and G. Pignatelli, "Business Capabilities Centric Enterprise Architecture", in: $P$. Bernus, G. Doumeingts, M. Fox (Ed.), Enterprise Architecture, Integration and Interoperability, vol. 326, Springer, Boston, USA, 2010, pp. 32-43.

[6] J. Beese, S. Aier, and R. Winter, "On the Role of Complexity for Guiding Enterprise Transformations", in: D. Aveiro, R. Pergl, M. Valenta (Ed.), Advances in Enterprise Engineering IX. EEWC, Lecture Notes in Business Information Processing, vol. 211, Springer, Cham, Switzerland, 2015, pp. 113-127.

[7] D. Beimborn, S. F. Martin, and U. Homann, "Capability-oriented modeling of the firm", in: IPSI Conference, Amalfi, Italy, 2005.

[8] A. Bharadwaj, O. A. El Sawy, P. A. Pavlou, and N. V. Venkatraman, "Digital Business Strategy: Toward a Next Generation of Insights", MIS Quarterly, 37 (2), 2013, pp. 471-482.

[9] E. Bouwers, J. Visser, and A. van Deursen, "Criteria for the evaluation of implemented architectures", in: IEEE International Conference on Software Maintenance (ICSM), Software Engineering Research Group, Delft, Netherlands, 2009, pp. 73-82.

[10] J. P. Brits, G. Botha, and M. Herselman, "Conceptual Framework for Modeling Business Capabilities", in: Proceedings of the Informing Science and IT Education Joint Conference, 2007, pp. 151-170.

[11] A. Freitag, F. Matthes, C. Schulz, and A. Nowobilska, "A method for business capability dependency analysis", in: International Conference on IT-enabled Innovation in Enterprise (ICITIE), Sofia, Bulgaria, 2011.

[12] W. Keller, "Using capabilities in enterprise architecture management", White Paper, Object Architects, 2009.

[13] C. Klinkmüller, A. Ludwig, B. Franczyk, and R. Kluge, "Visualising Business Capabilities in the Context of Business Analysis", in: W. Abramowicz, R. Tolksdorf (Ed.), Business Information Systems, vol. 47, Springer, Berlin/Heidelberg, Germany, 2010, pp. 242-253.

[14] J. K. Lankes, PhD thesis: "Metrics for Application Landscapes", Munich, Germany, 2008.
[15] Y. Malhotra, "Knowledge management and business model innovation", IGI Global, Hershey, PA, USA, 2001.

[16] Microsoft Corporation, "Microsoft Motion - Heat Mapping Tool," 2006. Retrieved from: http://blogs.microsoft.co.il/files/folders/2034/download. aspx [Accessed 9 April 2017].

[17] M. Mocker, "What is complex about 273 applications? Untangling application architecture complexity in a case of european investment banking", in: 42nd Hawaii International Conference on System Sciences, Hawaii, USA, 2009.

[18] A. W. Schneider, T. Reschenhofer, A. Schütz, and F. Matthes, "Empirical Results for Application Landscape Complexity", in: 48th Hawaii International Conference on System Sciences, Hawaii, USA, 2015.

[19] J. Scott, A. Cullen, and M. An, "The Anatomy Of A Capability Map", Forrester, 2010.

[20] M. Sykes and B. Clayton, "Surviving Turbulent Times: Prioritizing IT Initiatives Using Business Architecture", in: Advances in Computer Science: an International Journal, 2012, pp. 44-50.

[21] The Open Group, TOGAF Version 9.1 framework.

[22] U. Weber and V. Schmidtmann, "Differentiate. Der neue strategische Imperativ für den CIO”, 2008.

[23] J. Webster and R. T. Watson, "Analyzing the past to prepare for the future: Writing a literature review", MIS Quarterly, 26(2), 2002, pp. xiii-xxiii.

[24] F. Yilmaz, "Tool Support for Capability-Based Application Portfolio Management - Conceptualization, Prototype Implementation, and Evaluation", Masters Thesis, Technical University of Munich. 